\title{
Implementation of Computer Based Systems for Effective Deci- sions in Acceptance of Vikar
} \author{
Ulfa $^{2}$, K Khasanah', Imam Saputra ${ }^{2}$, Elvitrianim Purba ${ }^{7}$ \\ ${ }^{1}$ STT Pelita Kebenaran, Medan, Indonesia \\ ${ }^{2}$ STMIK Budi Darma, Medan, Indonesia \\ ${ }^{3}$ Universitas Methodist Indonesia, Medan, Indonesia \\ ${ }^{4}$ Sekolah Tinggi Keguruan dan Ilmu Pendidikan Biak, Papua, Indonesia \\ ${ }^{5}$ Sekolah Tinggi Teologi Sangkakala, Semarang, Indonesia \\ ${ }^{6}$ Universitas Padjadjaran, Bandung, Indonesia \\ ${ }^{7}$ AMIK STIEKOM Sumatera Utara, Rantauprapat, Indonesia \\ ${ }^{8}$ Universitas Negeri Medan, Medan, Indonesia \\ ${ }^{9}$ STMIK Indonesia, Jakarta, Indonesia \\ *Corresponding author E-mail: mesran.skom.mkom@gmail.com
}

Shinta Dharma Hardi ${ }^{1}$, Hukendik Hutabarat ${ }^{2}$, Benget Rumahorbo ${ }^{3}$, W Willem ${ }^{1}$, Mindo Judica Pangaribuan ${ }^{1}$, Kusman Sudibyo ${ }^{1}$, Andre Paulus Saleky ${ }^{4}$, Henry Kristian Siburian ${ }^{2}$, Fibry Jati Nugroho ${ }^{5}$, Nuning Kurniasih ${ }^{6}$, M Mesran $^{2 *}$, Maringan Sianturi ${ }^{2}$, Suardin Gaurifa ${ }^{1}$, Abdul Karim ${ }^{7}$,Janner Simarmata $^{8}$, Putri Ramadhani ${ }^{2}$, Kurnia

\begin{abstract}
The development of computer technology increasingly makes computers more popular in the community, so that it is easy to find computer usage in completing human work. The use of information technology is now increasingly developing with decision supportbased information systems that can help decision makers in providing better decision decisions. Likewise in the process of receiving Vikar in the GKPI church. Vicar candidates must go through the selection stage and at the final stage a decision-based support system is used to provide the right decision, the prospective Vikar who can be accepted in the GKPI. In this study, ARAS method is used which is expected to solve problems related to multi-criteria-based decision processing.
\end{abstract}

Keywords: Vikar Acceptance, ARAS Method, Computer Based System, DSS

\section{Introduction}

The Indonesian Protestant Christian Church (GKPI) is a place of worship for Christians in Indonesia. In Indonesia, there are several churches including the Batak Protestant Christian (HKBP), Indonesian Christian Huria (HKI), Pentecostal Church in Indonesia (GPDI). The leader in the GKPI church is a pastor, not only in the GKPI, all churches have a pastor's leadership, but before becoming a pastor must get a Vicar title. The process of receiving Vikar is not easy, because Vikar actually has to go through a period of education for several years. Although a Vikar has finished his education and is declared to have graduated, every Church must certainly do the selection first in the Vikar's acceptance.

Some conditions are set for the acceptance of Vikar in the GKPI, including education, administrative completeness, GPA, interviews, election results. To facilitate the processing of acceptance of Vicar, a computer-based information system is used that can process the data of prospective vikar, so that the results can be objective and effective. This information system is known as a decision support system, which can provide effective decisions on alternative alternatives that have mutual interest in several criteria with each other [1][2]. The development of decision support systems is very rapid, it can be in the form of desktop-based or webbased applications [3][4].
Sometime before, a lot of research was conducted related to decision support systems. Research has been done such as determining the best computer lecturer at STMIK Budi Darma by using ELECTRE method [5], determining the provision of school assistance using the WSM method [6], the process of selecting a laptop using Fuzzy Tahani [7], the selection of the best mechanics applies EXPROM II [8]

Among the decision support system methods above, there are also many methods that can be used in resolving those related to multicriteria, including the ARAS, VIKOR, COPRAS methods [9][12].

In this study, the method used in the process of receiving Vikar applies the Additive Ratio Assessment (ARAS) method which is expected to be able to provide effective results for decisions in receiving Vikar.

\section{Methodology}

Method of Additive Ratio Assessment (ARAS) was introduced by Zavadskas and Turksis. Method of Additive Ratio Assessment (ARAS) is able to solve complex problems using relatively simple 
comparisons. The work process in the Additive Ratio Assessment (ARAS) method is found in quantitative measurements and utility theory. In the ARAS method, the value of the utility function determines the relative efficiency of the alternatives above other alternatives. This utility function is directly proportional to the relative effect of the criteria value and weight value. Alternative utility values are determined by variations in comparison with the best ideal alternatives[10].

The following are the steps of the Additive Ratio Assessment (ARAS) method as follows [10][11]:

Step 1: Formation of a decision matrix

$X i j=\left[\begin{array}{cccc}X 11 & X 12 & \cdots & X 1 n \\ X 21 & X 22 & \cdots & X 2 n \\ \vdots & \vdots & \ddots & \vdots \\ X m 1 & X m 2 & \cdots & X m n\end{array}\right]$

Information

$$
\begin{aligned}
& \mathrm{m}=\text { Number of alternatives } \\
& \mathrm{n}=\text { Number of criteria }
\end{aligned}
$$

In the decision matrix the $X_{0 j}$ value is defined, if the $X_{0 j}$ value is unknown then it can be assumed that the value is obtained from the maximum value of the criterion (j) if the type of benefit, or the opposite of the minimum value if the type is cost.

Step 2: Normalize the decision matrix

If the Beneficial criteria:

$$
R i j=\frac{X i j}{\sum_{i=0}^{m} X i j}
$$

If the Non Beneficial criteria are normalized, follow the stages:

Stage-1: $X i j^{*}=\frac{1}{X i j}$

Stage-2: $R i j=\frac{X i j^{*}}{\sum_{i=0}^{m} X i j^{*}}$

$\mathrm{R}_{\mathrm{ij}}$ is a normalized matrix.

Step 3: Determine the normalized weighted matrix

$D=[d i j] m x n=r i j . w j$

Information

$\mathrm{W}_{\mathrm{j}}=$ the weight of criteria $\mathrm{j}$

Step 4: Determine the optimal function $\left(\mathrm{S}_{\mathrm{i}}\right)$

$S i=\sum_{j=1}^{n} d i j$

Step 5: Determine the degree of the utility $\left(\mathrm{U}_{\mathrm{i}}\right)$

$U i=\frac{S i}{S 0}$

The $U_{i}$ value is at the interval $[0,1]$, and the best value is the highest $U_{\mathrm{i}}$ value.

\section{Results and Discussion}

The application of information technology, in this case, the decision support system can provide objective results in data processing. The Vikar acceptance process was carried out in church congregation through several stages, including receiving files, checking the completeness of file administration, interviewing prospective vikar and the final determination stage whether the prospective vikar would be accepted as vikar. At the final stage, if the selection is still done without using a computer, the results of the decision will not be effective. This is because the process is still done manually without a computer. The existence of computer assistance in processing data can provide more effective results, far from subjective decisions. So that with the application of decision support methods can provide more effective results in vikar reception.

In determining the acceptance of vikar, alternative data is needed and the criteria used as a condition for receiving vikar. In table 1 , it is a criterion that is determined as a condition for receiving vikar.

Table 1. Criteria and Weight

\begin{tabular}{|c|l|c|c|}
\hline Criteria & \multicolumn{1}{|c|}{ Description } & Weight & Type \\
\hline $\mathrm{C}_{1}$ & Education & $30 \%$ & Benefit \\
\hline $\mathrm{C}_{2}$ & $\begin{array}{l}\text { Administrative } \\
\text { Completeness }\end{array}$ & $10 \%$ & Benefit \\
\hline $\mathrm{C}_{3}$ & GPA & $15 \%$ & Benefit \\
\hline $\mathrm{C}_{4}$ & Interview & $15 \%$ & Benefit \\
\hline $\mathrm{C}_{5}$ & Selection Test Value & $30 \%$ & Benefit \\
\hline
\end{tabular}

\begin{tabular}{|c|c|c|c|c|c|}
\hline & & & & & \\
\hline Alternauve & $\mathrm{C}_{1}$ & $\mathrm{C}_{2}$ & $\mathrm{C}_{3}$ & $\mathrm{C}_{4}$ & $\mathrm{C}_{5}$ \\
\hline Theodorus, S.Th $\left(\mathrm{A}_{1}\right)$ & $\mathrm{S} 1$ & Very Good & 3,05 & Good & 85 \\
\hline David, S.Th $\left(\mathrm{A}_{2}\right)$ & S1 & Very Good & 3,10 & Good & 84 \\
\hline Marpaung,S.Th $\left(\mathrm{A}_{3}\right)$ & S1 & Very Good & 3,25 & Good & 86 \\
\hline Sihombing, S.Th $\left(\mathrm{A}_{4}\right)$ & S1 & Good & 3,05 & $\begin{array}{l}\text { Very } \\
\text { Good }\end{array}$ & 84 \\
\hline Edy, S.Th $\left(\mathrm{A}_{5}\right)$ & $\mathrm{S} 1$ & Very Good & 3,00 & Good & 83 \\
\hline Elia, M.Th $\left(\mathrm{A}_{6}\right)$ & $\mathrm{S} 2$ & Very Good & 3,25 & Good & 81 \\
\hline Hermalela, S.Th $\left(\mathrm{A}_{7}\right)$ & $\mathrm{S} 1$ & Enough & 3,30 & Not Good & 83 \\
\hline Dewi, S.Th $\left(\mathrm{A}_{8}\right)$ & S1 & Very Good & 2,98 & Good & 85 \\
\hline Zulkarnain, M.Th $\left(\mathrm{A}_{9}\right)$ & S2 & Good & 3,40 & Enough & 88 \\
\hline Larose, S.Th $\left(\mathrm{A}_{10}\right)$ & S1 & Good & 3,15 & Good & 79 \\
\hline WagE, M.Th $\left(\mathrm{A}_{11}\right)$ & $\mathrm{S} 2$ & Good & 3,20 & Enough & 85 \\
\hline Bayu Togar, M.Th $\left(\mathrm{A}_{12}\right)$ & S2 & Very Good & 3,05 & $\begin{array}{l}\text { Very } \\
\text { Good }\end{array}$ & 90 \\
\hline Manalu, S.Th $\left(\mathrm{A}_{13}\right)$ & $\mathrm{S} 1$ & Good & 3,20 & Good & 79 \\
\hline Ferry, S.Th $\left(\mathrm{A}_{14}\right)$ & S1 & Very Good & 3,20 & $\begin{array}{l}\text { Very } \\
\text { Good } \\
\end{array}$ & 82 \\
\hline Andy, S.Th $\left(A_{15}\right)$ & $\mathrm{S} 1$ & Good & 3,15 & $\begin{array}{l}\text { Very } \\
\text { Good }\end{array}$ & 81 \\
\hline
\end{tabular}

The following list of candidates who become alternatives can be seen in table 1 .

From alternative candidates for vikar, there are linguistic data, namely education, administrative completeness, and interviews. In order to make the calculation easier, the data on these criteria are weighted as shown in table 3 and table 4 .

Table 3. The Weighting Educational Values (C1)

\begin{tabular}{|c|c|}
\hline Information & Weight \\
\hline S1 & 1 \\
\hline S2 & 2 \\
\hline S3 & 3 \\
\hline
\end{tabular}

Table 4. The weighting of Administrative Completeness (C2) and Interview (C4)

\begin{tabular}{|l|l|}
\hline Information & Weight \\
\hline Very Good & 4 \\
\hline Good & 3 \\
\hline Enough & 2 \\
\hline Not Good & 1 \\
\hline
\end{tabular}

From the weighting used in the table in table 2 based on tables 3 and 4 , the suitability rating is obtained as shown in table 5 .

Table 5. Match Rating

\begin{tabular}{|l|c|c|c|c|c|}
\hline \multicolumn{1}{|c|}{ Alternative } & $\mathrm{C}_{1}$ & $\mathrm{C}_{2}$ & $\mathrm{C}_{3}$ & $\mathrm{C}_{4}$ & $\mathrm{C}_{5}$ \\
\hline Theodorus, S.Th $\left(\mathrm{A}_{1}\right)$ & 1 & 4 & 3,05 & 3 & 85 \\
\hline David, S.Th $\left(\mathrm{A}_{2}\right)$ & 1 & 4 & 3,10 & 3 & 84 \\
\hline Marpaung,S.Th $\left(\mathrm{A}_{3}\right)$ & 1 & 4 & 3,25 & 3 & 86 \\
\hline Sihombing, S.Th $\left(\mathrm{A}_{4}\right)$ & 1 & 3 & 3,05 & 4 & 84 \\
\hline Edy, S.Th $\left(\mathrm{A}_{5}\right)$ & 1 & 4 & 3,00 & 3 & 83 \\
\hline Elia, M.Th $\left(\mathrm{A}_{6}\right)$ & 2 & 4 & 3,25 & 3 & 81 \\
\hline
\end{tabular}




\begin{tabular}{|l|c|c|c|c|c|}
\hline Hermalela, S.Th $\left(\mathrm{A}_{7}\right)$ & 1 & 2 & 3,30 & 1 & 83 \\
\hline Dewi, S.Th $\left(\mathrm{A}_{8}\right)$ & 1 & 4 & 2,98 & 3 & 85 \\
\hline Zulkarnain, M.Th $\left(\mathrm{A}_{9}\right)$ & 2 & 3 & 3,40 & 2 & 88 \\
\hline Larose, S.Th $\left(\mathrm{A}_{10}\right)$ & 1 & 3 & 3,15 & 3 & 79 \\
\hline WagE, M.Th $\left(\mathrm{A}_{11}\right)$ & 2 & 3 & 3,20 & 2 & 85 \\
\hline Bayu Togar, M.Th $\left(\mathrm{A}_{12}\right)$ & 2 & 4 & 3,05 & 4 & 90 \\
\hline Manalu, S.Th $\left(\mathrm{A}_{13}\right)$ & 1 & 3 & 3,20 & 3 & 79 \\
\hline Ferry, S.Th $\left(\mathrm{A}_{14}\right)$ & 1 & 4 & 3,20 & 4 & 82 \\
\hline Andy, S.Th $\left(\mathrm{A}_{15}\right)$ & 1 & 3 & 3,15 & 4 & 81 \\
\hline
\end{tabular}
The first step is to prepare a decision matrix based on table 5, can
be seen in table 6.

Table 6. Decision Matrix $\left(\mathrm{X}_{\mathrm{ii}}\right)$

\begin{tabular}{|c|c|c|c|c|}
\hline \multirow[t]{2}{*}{ Alternative } & $\mathrm{C}_{1}$ & $\mathrm{C}_{2}$ & $\mathrm{C}_{3}$ & $\mathrm{C}_{4}$ \\
\hline & Max & Max & Max & Max \\
\hline $\mathrm{A}_{0}$ & 2 & 4 & 3,4 & 1 \\
\hline$A_{1}$ & 1 & 4 & 3,05 & 3 \\
\hline $\mathrm{A}_{2}$ & 1 & 4 & 3,1 & 3 \\
\hline $\mathrm{A}_{3}$ & 1 & 4 & 3,25 & 3 \\
\hline $\mathrm{A}_{4}$ & 1 & 3 & 3,05 & 4 \\
\hline $\mathrm{A}_{5}$ & 1 & 4 & 3 & 3 \\
\hline $\mathrm{A}_{6}$ & 2 & 4 & 3,25 & 3 \\
\hline $\mathrm{A}_{7}$ & 1 & 2 & 3,3 & 1 \\
\hline $\mathrm{A}_{8}$ & 1 & 4 & 2,98 & 3 \\
\hline $\mathrm{A}_{9}$ & 2 & 3 & 3,4 & 2 \\
\hline $\mathrm{A}_{10}$ & 1 & 3 & 3,15 & 3 \\
\hline $\mathrm{A}_{11}$ & 2 & 3 & 3,2 & 2 \\
\hline$A_{12}$ & 2 & 4 & 3,05 & 4 \\
\hline $\mathrm{A}_{13}$ & 1 & 3 & 3,2 & 3 \\
\hline $\mathrm{A}_{14}$ & 1 & 4 & 3,2 & 4 \\
\hline $\mathrm{A}_{15}$ & 1 & 3 & 3,15 & 4 \\
\hline
\end{tabular}

For the value of $\mathrm{X} 0 \mathrm{j}$ is obtained from the best value in the criterion column (j). The next step uses equations 2,3 , and 4 containing the normalized matrix.

Table 7. Normalized matrix $\left(\mathrm{R}_{\mathrm{ij}}\right)$

\begin{tabular}{|c|c|c|c|c|}
\hline \multirow{2}{*}{ Alternative } & $\mathrm{C}_{1}$ & $\mathrm{C}_{2}$ & $\mathrm{C}_{3}$ & $\mathrm{C}_{4}$ \\
\cline { 2 - 5 } & $\mathrm{Max}$ & $\mathrm{Max}$ & $\mathrm{Max}$ & $\mathrm{Max}$ \\
\hline $\mathrm{A}_{0}$ & 0,0952 & 0,0714 & 0,0670 & 0,0889 \\
\hline $\mathrm{A}_{1}$ & 0,0476 & 0,0714 & 0,0601 & 0,0667 \\
\hline $\mathrm{A}_{2}$ & 0,0476 & 0,0714 & 0,0611 & 0,0667 \\
\hline $\mathrm{A}_{3}$ & 0,0476 & 0,0714 & 0,0641 & 0,0667 \\
\hline $\mathrm{A}_{4}$ & 0,0476 & 0,0536 & 0,0601 & 0,0889 \\
\hline $\mathrm{A}_{5}$ & 0,0476 & 0,0714 & 0,0591 & 0,0667 \\
\hline $\mathrm{A}_{6}$ & 0,0952 & 0,0714 & 0,0641 & 0,0667 \\
\hline $\mathrm{A}_{7}$ & 0,0476 & 0,0357 & 0,0651 & 0,0222 \\
\hline $\mathrm{A}_{8}$ & 0,0476 & 0,0714 & 0,0587 & 0,0667 \\
\hline $\mathrm{A}_{9}$ & 0,0952 & 0,0536 & 0,0670 & 0,0444 \\
\hline $\mathrm{A}_{10}$ & 0,0476 & 0,0536 & 0,0621 & 0,0667 \\
\hline $\mathrm{A}_{11}$ & 0,0952 & 0,0536 & 0,0631 & 0,0444 \\
\hline $\mathrm{A}_{12}$ & 0,0952 & 0,0714 & 0,0601 & 0,0889 \\
\hline $\mathrm{A}_{13}$ & 0,0476 & 0,0536 & 0,0631 & 0,0667 \\
\hline $\mathrm{A}_{14}$ & 0,0476 & 0,0714 & 0,0631 & 0,0889 \\
\hline $\mathrm{A}_{15}$ & 0,0476 & 0,0536 & 0,0621 & 0,0889 \\
\hline
\end{tabular}

Then calculating the normalized weighted matrix. At this stage the matrix has been normalized at times with weights on each criterion (equation 5), resulting in a weighted normalized matrix.

Table 8. The weighted of Normalization Matrix

\begin{tabular}{|c|c|c|c|c|}
\hline \multirow{2}{*}{ Alternatives } & $\mathrm{C}_{1}$ & $\mathrm{C}_{2}$ & $\mathrm{C}_{3}$ & $\mathrm{C}_{4}$ \\
\cline { 2 - 5 } & $\mathrm{Max}$ & $\mathrm{Max}$ & $\mathrm{Max}$ & $\mathrm{Max}$ \\
\hline $\mathrm{A}_{0}$ & 0,0286 & 0,0071 & 0,0101 & 0,0133 \\
\hline $\mathrm{A}_{1}$ & 0,0143 & 0,0071 & 0,0090 & 0,0100 \\
\hline $\mathrm{A}_{2}$ & 0,0143 & 0,0071 & 0,0092 & 0,0100 \\
\hline $\mathrm{A}_{3}$ & 0,0143 & 0,0071 & 0,0096 & 0,0100 \\
\hline $\mathrm{A}_{4}$ & 0,0143 & 0,0054 & 0,0090 & 0,0133 \\
\hline $\mathrm{A}_{5}$ & 0,0143 & 0,0071 & 0,0089 & 0,0100 \\
\hline $\mathrm{A}_{6}$ & 0,0286 & 0,0071 & 0,0096 & 0,0100 \\
\hline $\mathrm{A}_{7}$ & 0,0143 & 0,0036 & 0,0098 & 0,0033 \\
\hline $\mathrm{A}_{8}$ & 0,0143 & 0,0071 & 0,0088 & 0,0100 \\
\hline $\mathrm{A}_{9}$ & 0,0286 & 0,0054 & 0,0101 & 0,0067 \\
\hline $\mathrm{A}_{10}$ & 0,0143 & 0,0054 & 0,0093 & 0,0100 \\
\hline $\mathrm{A}_{11}$ & 0,0286 & 0,0054 & 0,0095 & 0,0067 \\
\hline $\mathrm{A}_{12}$ & 0,0286 & 0,0071 & 0,0090 & 0,0133 \\
\hline $\mathrm{A}_{13}$ & 0,0143 & 0,0054 & 0,0095 & 0,0100 \\
\hline
\end{tabular}

\begin{tabular}{|l|l|l|l|l|}
$\mathrm{A}_{14}$ & 0,0143 & 0,0071 & 0,0095 & 0,0133 \\
$\mathrm{~A}_{15}$ & 0,0143 & 0,0054 & 0,0093 & 0,0133 \\
\hline
\end{tabular}

The next process calculates the optimal function $(\mathrm{Si})$ using equation 6.

Table 9. Optimal Function Calculation Results $\left(\mathrm{S}_{\mathrm{i}}\right)$

\begin{tabular}{|c|c|}
\hline Alternative & $\mathrm{S}_{\mathrm{i}}$ \\
\hline $\mathrm{A}_{0}$ & 0,0793 \\
\hline $\mathrm{A}_{1}$ & 0,0596 \\
\hline $\mathrm{A}_{2}$ & 0,0595 \\
\hline $\mathrm{A}_{3}$ & 0,0604 \\
\hline $\mathrm{A}_{4}$ & 0,0609 \\
\hline $\mathrm{A}_{5}$ & 0,0590 \\
\hline $\mathrm{A}_{6}$ & 0,0735 \\
\hline $\mathrm{A}_{7}$ & 0,0496 \\
\hline $\mathrm{A}_{8}$ & 0,0594 \\
\hline $\mathrm{A}_{9}$ & 0,0704 \\
\hline $\mathrm{A}_{10}$ & 0,0567 \\
\hline $\mathrm{A}_{11}$ & 0,0692 \\
\hline $\mathrm{A}_{12}$ & 0,0783 \\
\hline $\mathrm{A}_{13}$ & 0,0569 \\
\hline $\mathrm{A}_{14}$ & 0,0627 \\
\hline $\mathrm{A}_{15}$ & 0,0605 \\
\hline
\end{tabular}

The final step is calculating the degree of the utility (Ui), using equation 7. In this final step, rank can be obtained from each candidate for Vikar.

Table 10. The Degree of the utility (Ui) and Rank

\begin{tabular}{|c|c|c|}
\hline Alternative & Ui & Rank \\
\hline $\mathrm{A}_{1}$ & 0,7507 & 9 \\
\hline $\mathrm{A}_{2}$ & 0,7497 & 10 \\
\hline $\mathrm{A}_{3}$ & 0,7610 & 8 \\
\hline $\mathrm{A}_{4}$ & 0,7674 & 6 \\
\hline $\mathrm{A}_{5}$ & 0,7432 & 2 \\
\hline $\mathrm{A}_{6}$ & 0,9269 & 15 \\
\hline $\mathrm{A}_{7}$ & 0,6253 & 11 \\
\hline $\mathrm{A}_{8}$ & 0,7481 & 3 \\
\hline $\mathrm{A}_{9}$ & 0,8878 & 14 \\
\hline $\mathrm{A}_{10}$ & 0,7149 & 4 \\
\hline $\mathrm{A}_{11}$ & 0,8718 & 1 \\
\hline $\mathrm{A}_{12}$ & 0,9870 & 13 \\
\hline $\mathrm{A}_{13}$ & 0,7168 & 7 \\
\hline $\mathrm{A}_{14}$ & 0,7898 & 5 \\
\hline $\mathrm{A}_{15}$ & 0,7626 & $\mathrm{~A}$ \\
\hline
\end{tabular}

After performing the calculation phase using the ARAS method, the results are $\mathrm{A} 12>\mathrm{A} 6>\mathrm{A} 9>\mathrm{A} 11>\mathrm{A} 14>\mathrm{A} 4>\mathrm{A} 15>\mathrm{A} 3>\mathrm{A} 1>$ A2> A8 $>$ A5 $>A 13>A 10>A 7$, and can get a list of prospective Vicar otherwise accepted taken from the 9 highest alternative values can be seen in table 11 .

Table 11. List of Candidates who received / not received \begin{tabular}{|c|c|c|}
\hline Alternative & Ui & Description \\
\hline
\end{tabular}

\begin{tabular}{|l|c|c|}
\multicolumn{1}{|c|}{ Alternative } & Ui & Description \\
\hline Theodorus, S.Th $\left(\mathrm{A}_{1}\right)$ & 0,987 & Received \\
\hline David, S.Th $\left(\mathrm{A}_{2}\right)$ & 0,9269 & Received \\
\hline Marpaung,S.Th $\left(\mathrm{A}_{3}\right)$ & 0,8778 & Received \\
\hline Sihombing, S.Th $\left(\mathrm{A}_{4}\right)$ & 0,7898 & Received \\
\hline Edy, S.Th $\left(\mathrm{A}_{5}\right)$ & 0,7674 & Received \\
\hline Elia, M.Th $\left(\mathrm{A}_{6}\right)$ & 0,7626 & Received \\
\hline Hermalela, $\mathrm{S} . T h\left(\mathrm{~A}_{7}\right)$ & 0,761 & Received \\
\hline Dewi, S.Th $\left(\mathrm{A}_{8}\right)$ & 0,7507 & Received \\
\hline Zulkarnain, $\mathrm{M} . T h\left(\mathrm{~A}_{9}\right)$ & 0,7497 & Not Received \\
\hline Larose, S.Th $\left(\mathrm{A}_{10}\right)$ & 0,7481 & Not Received \\
\hline WagE, M.Th $\left(\mathrm{A}_{11}\right)$ & 0,7432 & Not Received \\
\hline Bayu Togar, $\mathrm{M.Th}\left(\mathrm{A}_{12}\right)$ & 0,7168 & Not Received \\
\hline Manalu, S.Th $\left(\mathrm{A}_{13}\right)$ & 0,7149 & Not Received \\
\hline Ferry, S.Th $\left(\mathrm{A}_{14}\right)$ & 0,6253 & Not Received \\
\hline Andy, S.Th $\left(\mathrm{A}_{15}\right)$ & & \\
\hline
\end{tabular}

\section{Conclusion}

The results of the prospective vikar acceptance, obtained 7 alternatives that were propagated from 15 prospective vikar. The 7 alternatives can be accepted and appointed as vikar to the GKPI. The application of decision support systems to computer-based 
information systems can provide decisions for decision makers to produce better and more effective decisions.

\section{References}

[1] D. Siregar et al., "Multi-Attribute Decision Making with VIKOR Method for Any Purpose Decision," J. Phys. Conf. Ser., vol. 1019 no. 1,2018

[2] G.-H. Tzeng and J.-J. Huang, Multiple Attribute Decision Making Method And Applications. CRC Press, 2011.

[3] A. Yanie et al., "Web Based Application for Decision Support System with ELECTRE Method," J. Phys. Conf. Ser., vol. 1028, no. $1,2018$.

[4] S. H. Sahir et al., "The Preference Selection Index Method in Determining the Location of Used Laptop Marketing," Int. J. Eng. Technol., vol. 7, pp. 260-263, 2018.

[5] Mesran, G. Ginting, Suginam, and R. Rahim, "Implementation of Elimination and Choice Expressing Reality ( ELECTRE ) Method in Selecting the Best Lecturer ( Case Study STMIK BUDI DARMA )," Int. J. Eng. Res. Technol. (IJERT, vol. 6, no. 2, pp. 141-144, 2017.

[6] D. Handoko, M. Mesran, S. D. Nasution, Y. Yuhandri, and H Nurdiyanto, "Application Of Weight Sum Model (WSM) In Determining Special Allocation Funds Recipients," IJICS (International J. Informatics Comput. Sci., vol. 1, no. 2, pp. 31-35, 2017.

[7] S. H. Sahir, R. Rosmawati, and R. Rahim, "Fuzzy model tahani as a decision support system for selection computer tablet," Int. J. Eng. Technol., vol. 7, no. 2.9, pp. 61-65, 2018.

[8] M. Mesran, S. D. Nasution, S. Syahputra, A. Karim, and E. Purba, "Implementation of the Extended Promethee II in Upgrade Level of Mechanic," Int. J. Sci. Res. Sci. Technol., vol. 4, no. 2, pp. 125-130, 2018.

[9] Y. J. B. Parrangan et al., "The Implementation of VIKOR Method to Improve the Effectiveness of Sidi Learning Graduation," Int. J. Eng. Technol., vol. 7, pp. 264-267, 2018.

[10] Esra; and AyĢegül, “AIR CONDITIONER SELECTION PROBLEM WITH COPRAS AND ARAS METHODS," Manas $J$. Soc. Stud., vol. 5, no. 2, 2016.

[11] E. K. Zavadskas and Z. Turskis, "A new additive ratio assessment ( ARAS ) method in multicriteria decision - making," vol. 8619, 2011.

[12] Mesran, P. Ramadhani, A. Nasution, D. Siregar, Fadlina, and A. P. U. Siahaan, "Implementation of Complex Proportional Assessment Method in the Selection of Mango Seeds," Int. J. Sci. Res. Sci. Technol., vol. 3, no. 7, pp. 397-402, 2017. 